\title{
Triatoma mexicana Herrich-Schaeffer (Hemiptera: Reduviidae: Triatominae) Descripción de la Genitalia Externa del Macho y Morfología Externa de la Hembra
}

\author{
Paz María Salazar Schettino ${ }^{\bowtie}$, Gloria Elena Rojas Wastavino, José Santiago Rosales Piña, \\ Mauro Omar Vences Blanco \& Margarita Cabrera Bravo
}

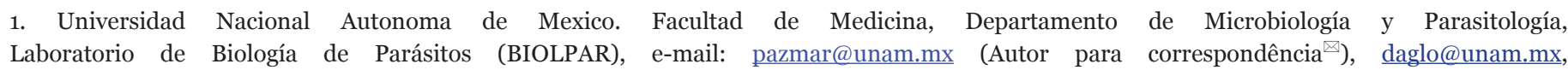
santirosapina@hotmail.com, mvences67@yahoo.com.mx, imay@unam.mx.

\section{EntomoBrasilis 6 (1): 68-73 (2013)}

Resúmen. Triatoma mexicana fue descrita originalmente en 1848 por Herrich-Schaeffer nombrándola Conorhinus mexicanus Herrich-Schaeffer. Actualmente se registra su hallazgo en los estados de Guanajuato, Hidalgo Querétaro y San Luis Potosí. Los estudios sobre su distribución espacial, índices entomológicos, presencia en el intradomicilio y peridomicilio, biología y riesgo de transmisión de Trypanosoma cruzi Chagas demuestran que es una especie importante desde el punto de vista de salud pública. Con el propósito de ampliar el conocimiento biológico sobre la especie, en el presente estudio se hace la descripción de la hembra así como la genitalia externa del macho, en ejemplares capturados en el estado de Guanajuato.

Palabras-Clave: Genitalia Externa del Macho; Guanajuato; México; Triatominae.

\section{Triatoma mexicana Herrich-Schaeffer (Hemiptera: Reduviidae: Triatominae) Description of the External Male Genitalia and External Morphology of the Female}

Abstract. Triatoma mexicana was originally described in 1848 by Herrich-Schaeffer, naming Conorhinus mexicanus Herrich-Schaeffer. Its finding is currently registered in the States of Guanajuato, Hidalgo, Querétaro, and San Luis Potosí. Studies about the spatial distribution, the entomological indices, it presence indoor an outdoor in houses, it biology and risk of transmission of Trypanosoma cruzi Chagas show that it is an important species from the point of view of public health. With the purpose of extending the biological knowledge of this species, the present study is the description of the female as well as the external male genitalia, in specimens captured in the State of Guanajuato.

Keywords: External Male Genitalia; Guanajuato; Mexico; Triatominae.

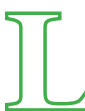
a subfamilia Triatominae (Hemiptera: Reduviidae) se divide en cinco tribus, 15 géneros y 140 especies (SCHOFIELD \& GaLVão 2009). En México, se han reportado 32 especies transmisoras de Trypanosoma cruzi Chagas, agente causal de la enfermedad de Chagas (Martínez et al. 2006), aunque se han reportado reportado otros arreglos taxonómicos, como el descrito por GaLVão et al. 2003. Veinticinco especies pertenecen al género Triatoma, dos especies al género Panstrongylus y una especie de cada uno de los siguientes géneros: Belminus, Dipetalogaster, Eratyrus, Paratriatoma y Rhodnius. Trece géneros se encuentran asociados a la vivienda humana, dos en el intradomicilio y once en el peridomicilio entre estos últimos se ubica a Triatoma mexicana (Herrich-Schaeffer) (VIDAL et al. 2000, SAlazAr et al. 2010).

El primer registro de T. mexicana lo realizó HERRICH-SCHAEFFER, en 1848, para ello describió un ejemplar macho denominándolo Conorhinus mexicanus HerRich-Schaeffer. Aproximadamente, 100 años después, el Dr. Luis Mazzotti en 1940, realizó a una amplia descripción de un ejemplar macho, capturado en Metztitlan, en el estado de Hidalgo, en el que muestra las diferencias entre el ejemplar estudiado y Triatoma phyllosoma Burmeister, especie con la que habían encontrado semejanzas STR̊L en 1859 y Champion en 1901, sin embargo, aunque la descripción de Herrich-Schaeffer en 1848 es breve, la descripción realizada por Mazzotтi (1940) se apoya en la descrita por este último entomólogo. LENT \& WigodZinsKY (1979), realizan una exhaustiva descripción externa de un ejemplar macho capturado en la localidad de Ayutla, situada en el Municipio de Arroyo Seco, en el estado de Querétaro.

Actualmente $T$. mexicana se encuentra distribuida en cuatro estados: Guanajuato, Hidalgo, Querétaro y San Luis Potosí (LeNT \& WigodZinsKY 1979; Zárate \& Zárate 1985; VidAl et al. 2000; Villagrán et al 2008). El hallazgo de los transmisores se ha realizado tanto en el intradomicilio como en el peridomicilio, la infección natural con T. cruzi se reportó por primera vez en los estados de Guanajuato e Hidalgo (VIDAL et al. 200o). En el estado de Guanajuato su presencia y distribución ha sido extensamente registrada, fue capturada en 34 de los 46 municipios que componen el estado, entre los 900 y $2200 \mathrm{msnm}$, con 2,9\% $(29 / 1009)$ de infección natural y con un índice de dispersión de $18,9 \%$ con respecto a las otras tres especies que se reportan en el estado (4,3\% Triatoma barberi Usinger, 4,7\% Triatoma pallidipennis Stål y 1,1\% Triatoma longipennis Usinger). El hallazgo fue en paredes y dormitorios del intradomicilio, $56 \%$ y $25 \%$, respectivamente. T. mexicana representa, en el estado, un alto riego para la transmisión de $T$. cruzi, agente causal de la enfermedad de Chagas (LópEz et al. 2005).

Otros autores reportan, en el mismo estado, su hallazgo principalmente en el peridomicilio $(151 / 165)$ en las paredes externas de las casas (54\%) y bajo las cercas o vallas de piedras, lugar donde llevan a cabo su ciclo de vida ya que se encontraron los cinco estadios ninfales y los adultos, coincidiendo con el comportamiento de T. pallidipennis en el estado de Morelos (BAUTISTA et al 1999), en este estudio el mayor porcentaje de ejemplares capturados realizó durante la primavera y la mayoría 
fueron adultos (137/165), con un índice de infección natural de entre 2,6 y 4,7\%, mientras que el índice de infestación osciló entre 4,2 y 18,1\%(SALAZAR et al. 2007).

En investigaciones realizadas en el estado de Hidalgo, $T$. mexicana se capturó en el municipio de Tasquillo, en la localidad de Caltimacán, la tasa de infección fue de 6,9\%, los aislados de T. cruzi obtenidos demostraron negatividad en ratón; el índice de infestación fue de 11,4\% (BECERRIL et al. 2007). En otro estudio realizado en diez localidades del municipio de Meztitlan, Hidalgo, con el propósito de estudiar los índices entomológicos y las características de la vivienda se detectaron tres de cinco localidades infestadas con $T$. mexicana, con un índice de infestación de 7,7\% a 20\%, con un índice de hacinamiento de 2.6 chinches por casa y con un índice de colonización de $0 \%$. También se detectó que su presencia predomina en viviendas construidas con paredes con acabados planos de yeso y cemento. (BECERRIL et al. 2010), comportamiento observado por SALAZAR et al (2007) y por BAUTISTA et al. (1999) en T. pallidipennis.

En una investigación realizada en el estado de Querétaro, T. mexicana se encontró en seis de los siete municipios estudiados, $8 \%$ en el intradomicilio (40/473) y $92 \%$, peridomicilio (40/473) de las cuatro especies encontradas (Triatoma gerstaeckeri Stål, T. pallidipennis y Triatoma dimidiata Latreille), T. mexicana mostró $28,7 \%$ de infección natural, sólo esta última especie se encontró en ambos ecotopos (VILLAGRán et al.2008).

Con el propósito de estudiar la biología de T. mexicana, en estudios realizados en condiciones de laboratorio y a tres diferentes condiciones de temperatura y humedad se comprobó que este transmisor tiene un período de incubación que oscila entre los 16 y 28 días, el desarrollo de huevo a adulto es de 255 días, a $30^{\circ} \mathrm{C}$ y $75 \%$ de HR, el tiempo de alimentación es sobre los 14 minutos y el tiempo de defecación es de 18 minutos en promedio, lo que lo hace un mal transmisor de T. cruzi (MARTínez-IBARRA et al. 2008). En otro estudio sobre la biología del desarrollo de T. mexicana, el ciclo completo se llevó a cabo en $338,66 \pm 35$ días a $27^{\circ} \mathrm{C}$ y $66 \%$ de HR (BECERRIL et al. 2008).

Con la finalidad de aportar nuevos datos sobre esta especie se realizó un estudio sobre la genitalia del macho de T. mexicana, ya que las estructuras fálicas son útiles para caracterizar y separar especies, géneros tribus y poblaciones. Según los conceptos descritos por JURBERg et al. (1998) sobre la genitalia externa de la tribu Triatomini, ésta se ubica entre los segmentos octavo y noveno del conexivo, este último llamado también pigóforo y se localiza en la región ventral. Los elementos que se usan para estudiar la genitalia de los triatominos incluyen estructuras como los parámeros, el pigóforo, falo, el proceso del endosoma, la vesica Los parámeros son apéndices articulados, de forma enrollada, con el ápice curvado y están localizados lateralmente a la abertura genital. Internamente la base está unida al falo a través del proceso capitata. El pigóforo está dividido internamente, en dos áreas, por una membrana llamada diafragma, la zona superior es la cavidad visceral. El falo está compuesto por dos partes diferentes, una es la basal, llamado aparato articular, unido al cuerpo del insecto, la otra es la apical, extensible y llamada edeago. La parte basal tiene el musculo protractor y retractor del falo, insertado a través de tres procesos capitata, con forma de hongo y unido al pigóforo. El proceso capitata 1 está unido al parámero, el proceso capitata 2 al pigóforo y el proceso capitata 3 al diafragma. El endosoma está formado por una membrana elástica localizada en el centro del edeago que durante la copulación. Durante la copulación está inflada dentro del cuerpo de la hembra. La vesica es una sola estructura localizada en la parte eversible del endosoma (JURBERG et al. 1998).

El falo de los ejemplares de la tribu Triatomini se caracteriza por ocupar toda el área inferior del edeago, el soporte del falosoma es largo, con un apéndice dividido en dos brazos que en algunos casos pueden unirse. La vesica es impar y el proceso del endosoma puede tener ornamentaciones Varios estudios previos han reportado la genitalia externa de machos de diferentes especies capturadas en México (Lent \& JuRberg 1982, 1985, 1987)

El objetivo del presente estudio es describir la genitalia del macho de la especie T. mexicana y dar a conocer las medidas externas de las hembras en ejemplares capturados en tres municipios del estado de Guanajuato

\section{MATERIAL Y MÉTODOS}

Se utilizaron ejemplares machos y hembras de la especie $T$. mexicana, identificados con las claves de LENT \& WygODZINSKY (1979), capturados en tres municipios ubicados al noreste del estado de Guanajuato, denominados: Victoria, Santa Catarina y Tierra Blanca. Los tres municipios están localizados entre los $20^{\circ} 42^{\prime}$ y $21^{\circ} 13^{\prime}$ de latitud norte y entre $100^{\circ} \mathrm{O} 2^{\prime}$ y $100^{\circ} 17^{\prime}$ de longitud oeste, entre 1593 y 1844 msnm (SALAZAR et al. 2007). Los ejemplares fueron mantenidos en condiciones ambientales de temperatura y humedad.

La captura de los insectos se llevó a cabo en el intradomicilio y el peridomicilio de las viviendas; sin embargo, todos los ejemplares adultos fueron capturados en el peridomicilio, en diversos sitios de la vivienda, como las paredes exteriores de los patios y en las bardas de piedra.

Para realizar las mediciones y la descripción de la hembra, se usaron 15 hembras, las observaciones fueron hechas en un microscopio estereoscópico Carl Zeiss S20193, con reglilla (1mm) con aumento de 0.8 en el Laboratorio de Acarología de la Facultad de Ciencias de la Universidad Nacional Autónoma de México.

Para describir la genitalia del macho, se usaron 7 machos, el IX segmento que conforma la cápsula genital fue sometido a un proceso de ablandamiento con $\mathrm{KOH}$ al $10 \%$, a temperatura ambiente durante una hora, posteriormente, se extrajo el falo con ayuda de agujas entomológicas, pinzas de punta fina y un microscopio estereoscópico. Las estructuras se colocaron nuevamente en la solución de $\mathrm{KOH}$ por 4 horas a temperatura ambiente, más tarde se neutralizaron con ácido acético al 10\%; se rotularon y se conservaron en tubos de ensayo con glicerina. Se retiró el proceso del pigóforo de la cápsula genital y se disecó el falo, se extrajeron la vesica, el proceso del endosoma, el falosoma y el soporte del falosoma. Esta parte de la investigación se realizó en dos tiempos, las fotos de las figuras 1-3-4 se tomaron con una cámara Cannon EOS Rebel Tii, con lentes Macro Nikkor HM$12 \mathrm{X}$ con iluminación transmitida y tangencial, en un microscopio estereoscópico Carl Zeiss, a una distancia focal de $23 \mathrm{~mm}$. Las fotos 2-5-6 se tomaron con una cámara Nikon E990, con reglilla en los oculares.

\section{RESULTADOS Y DISCUSIÓN}

En el presente estudio se midieron las estructuras externas de la hembra de $T$. mexicana con el fin de ampliar el conocimiento y proporcionar datos para el correcto diagnóstico de la especie, ya que los datos conocidos acerca de esta especie estaban basados sólo en la descripción de los machos.

La hembra de $T$. mexicana presenta el mismo patrón de coloración que los machos, es decir, negro con marcas amarillas en el cuello, corion y conexivo. Los hemielitros son cortos y no exceden más allá de la base del séptimo segmento abdominal. Abdomen fuertemente amplio con hemielitros dejando porciones laterales de los urotergitos expuestos (Figura 1).

El promedio de las medidas realizadas en los ejemplares de las hembras de T. mexicana se muestran en la Tabla 1.

Descripción de la genitalia del macho de $T$. mexicana. La genitalia de los machos se localiza en el $8^{\circ}$ y $9^{\circ}$ segmento en la 
cara abdominal o ventral del insecto, región que recibe el nombre de Pigóforo (Figura 2), se localiza además otra estructura llamada proceso medio de pigóforo el cual es de forma triangular delgado y corto, mide $416 \mu$, con presencia de pelos largos (Figura 3). Cuenta con órganos accesorios para la copula llamados Parámeros (Figura 4), éstos son de forma cilíndrica arqueados, cubiertos por pelos medianos y con una proyección en la región apical.

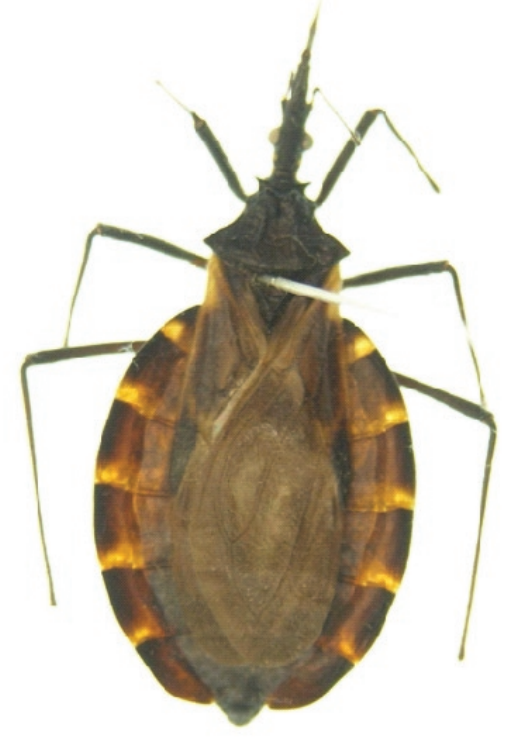

Figura 1. Ejemplar hembra de Triatoma mexicana (barra=25.4 mm). BIOLPAR.

Tabla 1. Medidas de las estructuras morfológicas de la hembra de Triatoma mexicana.

\begin{tabular}{|c|c|c|}
\hline & didas & Hembras (mm) \\
\hline \multicolumn{2}{|c|}{ Longitud Total } & 25,438 \\
\hline \multicolumn{2}{|c|}{ Ancho del abdomen } & 10,427 \\
\hline \multicolumn{2}{|c|}{ Longitud de la cabeza } & 4,328 \\
\hline \multicolumn{2}{|c|}{ Región Anteocular } & 2,498 \\
\hline \multicolumn{2}{|c|}{ Región Postocular } & 0,79 \\
\hline \multicolumn{2}{|c|}{ Synthlipsis } & 0,845 \\
\hline \multicolumn{2}{|c|}{ Longitud del Pronoto } & 3,928 \\
\hline \multicolumn{2}{|c|}{ Ancho del Pronoto } & 5,57 \\
\hline \multirow[t]{3}{*}{ Rostro } & 1 & 1,755 \\
\hline & 2 & 2,54 \\
\hline & 3 & 0,844 \\
\hline \multirow[t]{4}{*}{ Antenas } & 1 & 0,962 \\
\hline & 2 & 3,447 \\
\hline & 3 & 2,353 \\
\hline & 4 & 1,473 \\
\hline \multicolumn{2}{|c|}{ Fémur } & 8,351 \\
\hline \multicolumn{2}{|c|}{ Ancho del Ojo } & 1,66 \\
\hline \multicolumn{2}{|c|}{ Ancho del ocelo } & 0,388 \\
\hline \multicolumn{2}{|c|}{ Ancho del Cuello } & 1,175 \\
\hline \multicolumn{2}{|c|}{ Ancho del Escutelo } & 2,068 \\
\hline
\end{tabular}

Dentro del pigóforo se localiza el falo, constituido de dos partes, el edeago y el aparato articular, éste se encuentra fijo al pigóforo por medio de los procesos capitatos localizados en el ápice del aparato articular, donde se insertan músculos pro-tractores y retractores del falo que permiten que este salga del pigóforo efectuando una rotación de $180^{\circ}$. El aparato articular es una placa de tres ramas con aspecto de $\mathrm{Y}$ invertido está conformado por la expansión media de la placa basal, el proceso del gonoporo, proceso del capitato, el puente basal y la placa basal (Figura 5).

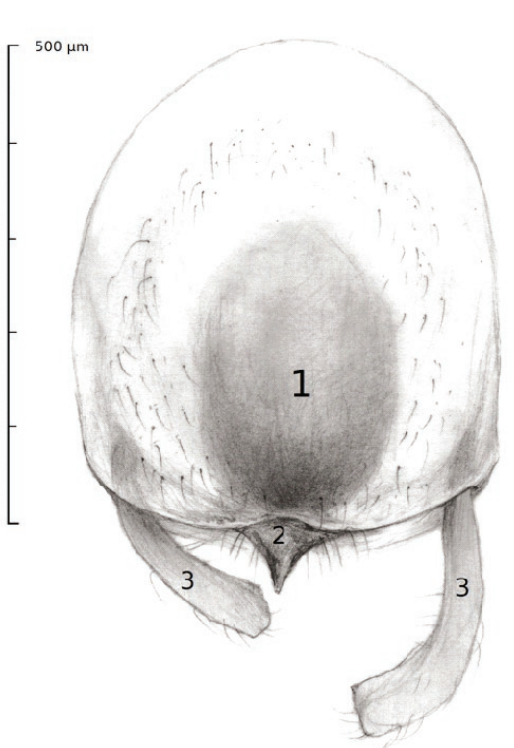

Figura 2. Estructura del falosoma. Pigóforo vista ventral de la genitalia del macho de Triatoma mexicana 1.- Falosoma. 2.- Proceso medio del pigóforo. 3.- Parámeros. BIOLPAR.

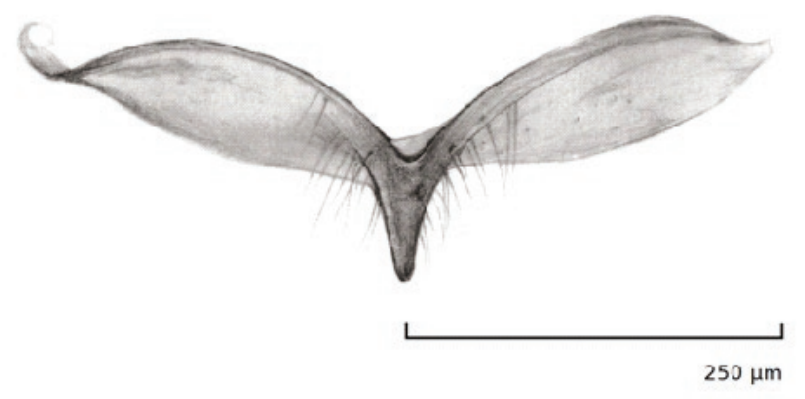

Figura 3. Proceso medio del pigóforo de la genitalia del macho de Triatoma mexicana. BIOLPAR.

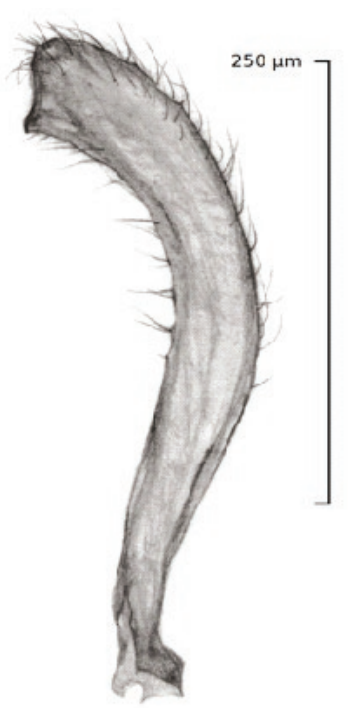

Figura 4. Parámero de la genitalia del macho de Triatoma mexicana. Laboratorio de Biología de parásitos

El edeago es una estructura de forma globosa constituido en su parte externa por el falosoma y la conjuntiva y en la interna por el endosoma, el proceso del Endosoma, la vesica y el soporte del falosoma.

El falosoma está constituido por una placa laminar que sirve de soporte al endosoma, en su parte basal es amplio y hacia su parte apical acuminado (disminuye gradualmente y termina en punta), con una pequeña abertura. En la cara interna del falosoma se encuentra adherido el soporte del falosoma, estructura en forma 
T deh de horqueta tubular hueca cuyos brazos se encuentran unidos en su parte basal y se observan separados en su ápice, mide $468 \mu$ (Figura 6).

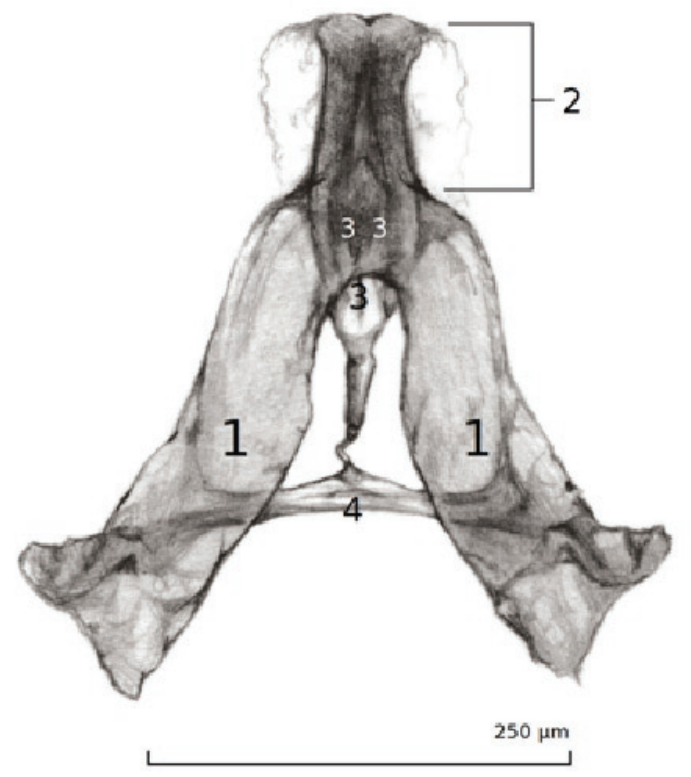

Figura 5. Vista dorsal del aparato articular de la genitalia de macho de Triatoma mexicana. 1.-placa basal; 2.- extensión mediana de la placa basal; 3.- proceso del gonoporo; 4.- puente basal. BIOLPAR.

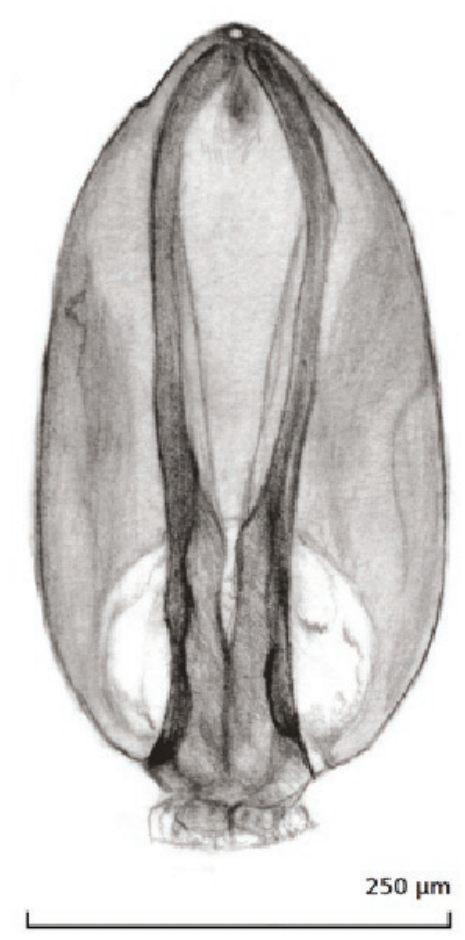

Figura 6. Falosoma y soporte del falosoma de la genitalia del macho de Triatoma mexicana. BIOLPAR.

El endosoma es membranoso con unas estructuras quitinizadas situadas lateralmente y cubiertas de espinas en la región del ápice (Figura 7) y, una estructura impar llamada vesica, la vesica presenta un borde superior elevado y la parte lateral es membranosa, vista de forma lateral presenta una forma ovoide, mide $335 \mu$ (Figura 8).

La especie $T$. mexicana, ha sido incluida en el complejo Phyllosoma, basándose en estudios taxonómicos usando la secuencia mtCytB, los resultados mostraron una fuerte asociación entre T. mexicana y Triatoma bassolsae Alejandre, con respecto a los otros géneros que componen este complejo (MarTínez et al. 2006). Mientras que Schofield \& Galvão (2009) la ubican en el grupo Rubrofasciata, complejo Phyllosoma y subcomplejo Phyllosoma (=Meccus) al proponer una nueva clasificación. Sin embargo, estudios relacionados a la biología de las especies que componen el complejo muestran coincidencias y diferencias cuando se comparan con el comportamiento biológico de $T$. mexicana, en investigaciones realizadas bajo las mismas condiciones de laboratorio. Así T. mexicana presenta un tasa de eclosión del 60\%, comparada con el 70\% de las especies del complejo; el promedio de tiempo para alcanzar la etapa adulta fue más corto en $T$ mexicana coincidiendo con los tiempos de Meccus picturatus Usinger y Meccus pallidipennis Stål; el promedio del tiempo de desarrollo en los ejemplares alimentados en conejo fue sobre los 255 días igual que en Meccus bassolsae Alejandre, pero diferente para Meccus phyllosomus Burmeister y $M$. pallidipennis; el promedio de ingesta de sangre antes de la muda fue más alto cuando se usó un protocolo con temperatura y humedad relativa bajo, coincidiendo con los resultados obtenidos para M. pallidipennis; la mortalidad fue más alta en las ninfas de primero y segundo estadio, coincidiendo con todas las especies del complejo; el promedio del tiempo de defecación fue sobre los 18 minutos en T. mexicana a diferencia de todas las especies del complejo las cuales presentan un tiempo de menos de 10 minutos, comportamiento que las hace diferentes y por lo tanto mejores transmisores de $T$. cruzi, razón por la cual los autores no la consideran como una especie del complejo Phyllosoma (MARTÍNEZ-IBARRA et al. 2008). El mismo autor comprobó el aislamiento reproductivo de T. mexicana y las especies del complejo Phyllosoma, al realizar cruzas parentales, que aunque las hembras ovipositaron, ninguno de los estadios ninfales logró alcanzar el estado adulto (MARTínEZ-IBARRA et al. 2011).

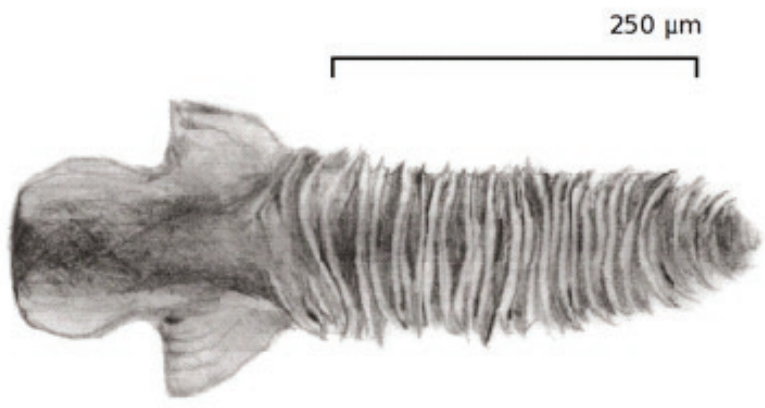

Figura 7. Proceso del endosoma de la genitalia del macho de Triatoma mexicana. BIOLPAR.

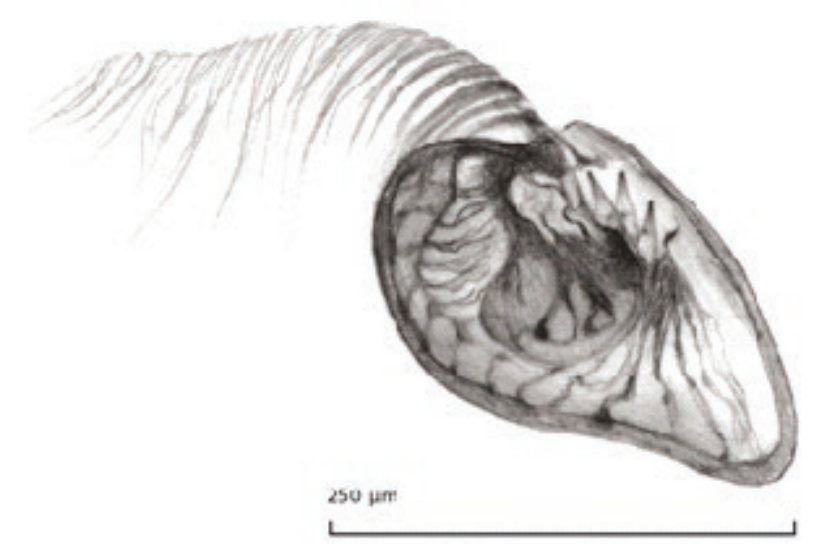

Figura 8. Vesica de la genitalia del macho de Triatoma mexicana. BIOLPAR. 
Con respecto a las medidas de la hembra estas también difieren con aquellas especies del complejo ya que en esta investigación la longitud total fue de 25,43 mm; LENT \& Wigodzinsky (1979) reportan 30-37 $\mathrm{mm}$ para $T$. longipennis, $34 \mathrm{~mm}$ para Triatoma mazzottii Usinger, 32-35 mm para T. pallidipennis, 32-33mm para $T$. phyllosoma y 29-39 $\mathrm{mm}$ para $T$. picturata . T. mexicana muestra diferentes medidas cuando es comparada con $T$. longipennis, al coincidir en la medida de algunas estructuras, así, la longitud de la cabeza es $4,32 \mathrm{~mm}$, contra $5,5 \mathrm{~mm}$ de $T$. longipennis; la longitud del segundo segmento rostral es de $2,54 \mathrm{~mm}$ contra $3,2 \mathrm{~mm}$ en $T$. longipennis; en el ancho del abdomen en $T$. mexicana mide 10,42mm y en $T$. longipennis es de 13,8 mm (MARTÍNEZ-IBARRA et al. 2006).

Los ejemplares usados para la descripción de la genitalia del macho para este trabajo fueron capturados en una misma zona, por lo que no fue posible evaluar las diferencias que pudieran presentarse en poblaciones de una misma especie, capturadas en diferentes lugares geográficos.

\section{AGRADECIMIENTOS}

Al personal de vectores de la Jurisdicción Sanitaria 2, San Miguel de Allende, Guanajuato, por su participación en el trabajo de campo. Al Dr. Ignacio Vázquez Rojas del Laboratorio de Acarología, Facultad de Ciencias, UNAM, por las facilidades para realizar las medidas morfológicas. A los biólogos Armando Zepeda Rodríguez y Francisco G. Pasos Nájera del Laboratorio de Microscopia Electrónica, del Departamento de Biología Celular y Tisular, de la Facultad de Medicina de la UNAM, por el apoyo en la toma de las fotografías y medidas de las mismas.

\section{REFERENCIAS}

Bautista N.L., G. García de la Torre, I. De Haro \& P.M. SalazarSchettino, 1999. Importance of Triatoma pallidipennis (Hemiptera: Reduviidae) as a vector of Trypanosoma cruzi (Kinetoplastida:Trypanosomatidae) in the state of Morelos, Mexico and possible ecotope, Journal of Medical Entomology, 36: 233-235.

Becerril M.A., A. Aguilar \& J.L. Imbert-Palafox, 2008. Biologic Development of Triatoma mexicana (Herrich-Schaeffer 1848) (Hemiptera: Reduviidae: Triatominae) under Laboratory Conditions. International Journal of Tropical Medicine, 3: 85-90.

Becerril M.A., E. Rangel, J. L. Imbert, J. Gómez, \& H. Figueroa, 2007. Human infection risk of transmission of Chagas disease in Hidalgo state, Mexico. The American Journal of Tropical Medicine and Hygiene, 76: 318-323.

Becerril M.A., V. Angeles-Pérez, J. Noguez-García \& J.L.ImbertPalafox, 2010. Riesgo de Transmisión de Trypanosoma cruzi en el Municipio de Metztitlán, Estado de Hidalgo, México, Mediante la Caracterización de Unidades Domiciliares y sus Índices Entomológicos. Neotropical Entomology, 39: 810817.

Galvão, C., R. Carcavallo, D. Da Silva Rocha \& J Jurberg. 2003. A checklist of the current valid species of the subfamily Triatominae Jeannel, 1919 (Hemiptera, Reduviidae) and their geographical distribution, with nomenclature and taxonomic notes. Zootaxa, 202: 1-36.

Jurberg, J., H. Lent \& Cleber Galvão, 1998. The male genitalia and its importance in taxonomy, p. 85-106. In: Carcavallo, R.U., I. Galindez Girón, J. Jurberg \& H Lent (Eds.), Atlas of Chagas' disease vectors in the Américas, vol I, Fiocruz, Rio de Janeiro, 393p.

Lent, H. \& J. Jurberg, 1982. A genitália externa dos machos no complex Triatoma phyllosoma (Burn.), espécies mexicanas transmissoras da doença de Chagas (Hemiptera, Reduviidae). Memorias do Instituto Oswaldo Cruz, 77: 299-317.

Lent, H. \& J. Jurberg, 1985. Sobre a variação intra-especifica em Triatoma dimidiata (Latreille) e Triatoma infestans (Klug) (Hemiptera, Reduviidae). Memorias do Instituto Oswaldo
Cruz, 80: 285-299.

Lent, H. \& J. Jurberg, 1987. A genitalia externa dos machos de sete espécies de Triatoma Laporte, 1832 da região neártica (Hemiptera, Reduviidae, Triatominae). Memorias do Instituto Oswaldo Cruz, 82: 227-246.

Lent, H. \& P. Wygodzinsky, 1979. Revision of the Triatominae (Hemiptera, Reduviidae) and their significance as vector of Chagas' disease. Bulletin of the American Museum of Natural History, 163: 123-520.

López J., F. González, P.M. Salazar, J. Gallaga, E. Ramírez, J. Martínez- Mendes, V. Sánchez, A. Townsend, \& J. Ramsey, 2005. Fine-scale predictions of distributions of Chagas disease vectors in the state of Guanajuato, Mexico, Journal of Medical Entomology, 42: 1068-1081.

Martínez F., G. Villalobos, A. Cevallos, P. De la Torre, J. Laclette, R. Alejandre \& B Espinoza, 2006. Taxonomic study of the phyllosoma complex and other triatomine (Insecta: Hemiptera: Reduviidae) species of epidemiological importance in the transmission of Chagas disease: Using ITS-2 and mtCytB sequences. Molecular Phylogenetics and Evolution, 41: 279-287.

Martínez-Ibarra J.A., P.M. Salazar, M. Solorio, M. Cabrera, M. Novelo, M. Vences, J. Montes \& B. Nogueda, 2008. Influence of temperature and humidity on the biology of Triatoma mexicana (Hemiptera: Reduviidae: Triatominae) under laboratory conditions. Memorias do Instituto Oswaldo Cruz, 103: 719-723.

Martínez-Ibarra J.A., Y. Guillén, I. Delgadillo, F. Zumaya, G. Rocha, P. Salazar, R. Alejandre, G. Villalobos \& B. Nogueda, 2011. Biological and genetic aspects of crosses between phylogenetically close species of mexican triatomines (Hemiptera: Reduviidae). Journal of Medical Entomology, 48: 705-707.

Martínez-Ibarra, J.A., N. Bárcenas, J. Nápoles, B. Nogueda \& M. Rodríguez, 2006. Diferencias métricas entre poblaciones de Meccus longipennis (Usinger) (Hemiptera: Reduviidae) en el occidente de México. Folia Entomologica Mexicana, 45: 8390.

Mazzotti, L., 1940. Una especie rara de Triatoma. Triatoma mexicana (H. Schaeffer, 1848). Medicina. Revista Mexicana, 359: 127-131.

Salazar, P.M., G. Rojas, M. Cabrera, M. Bucio, J. MartínezIbarra, M.C. Monroy, A. Rodas, Y. Guevara, M. Vences, A. Ruiz \& E. Torres, 2010. Revisión de 13 especies de la familia Triatominae (Hemiptera: Reduviidae) vectores de la enfermedad de Chagas, en México, Journal of the Selva Andina Research Society, 1: 57-80.

Salazar, P.M., J. Rosales, G. Rojas, M. Cabrera, M. Vences \& J. López, 2007. Triatoma mexicana (Hemiptera: Reduviidae) in Guanajuato, Mexico: House infestation and seasonal variation. Memorias do Instituto Oswaldo Cruz, 102: 803807.

Schofield, C.J. \& C. Galvão, 2009. Classification, evolution and species groups within the Triatominae. Acta Tropica, 110: 88100.

Vidal, V., S. Ibáñez \& C. Martínez, 200o. Infección Natural de chinches Triatominae con Trypanosoma cruzi asociadas a la vivienda humana en México. Salud Pública México, 42: 496503.

Villagrán, M.E., C. Marín, A. Hurtado, M. Sánchez-Moreno \& J.A. de Diego, 2008. Natural infection and distribution of triatomines (Hemiptera: Reduviidae) in the state of Querétaro, Mexico. Transactions of the Royal Society of Tropical Medicine and Hygiene, 102: 833-888.

Zárate L. \& R. Zárate, 1985. A checklist of the Triatominae (Hemiptera: Reduviidae) of Mexico, International Journal of Entomology, 27: 102-127.

Recebido em: 04/o9/2012

Aceito em: 26/12/2012 
Como citar este artigo:

Schettino, P.M.S., G.E.R. Wastavino, J.S.R. Piña, M.O.V. Blanco, M.C. Bravo, 2013. Triatoma mexicana Herrich-Schaeffer (Hemiptera: Reduviidae:

Triatominae) Descripción de la Genitalia Externa del Macho y Morfología Externa de la Hembra. EntomoBrasilis, 6(1): 68-73.

Acessível em: http://www.periodico.ebras.bio.br/ojs/index.php/ebras/article/view/275. doi:10.12741/ebrasilis.v6i1.275

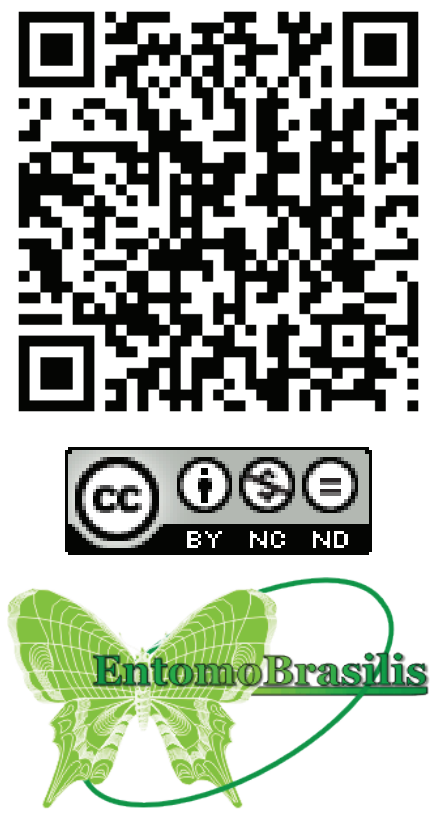

